\title{
Establishing a pre-clinical growing animal model to test a tissue engineered valved pulmonary conduit
}

\author{
Walter Knirsch $^{1,2 \#}$, Bernard Krüger ${ }^{3,4 \#}$, Thea Fleischmann ${ }^{5}$, Alexandra Malbon ${ }^{6}$, Miriam Lipiski $^{5}$, Frithjof \\ Lemme $^{2,7}$, Mareike Sauer $^{5}$, Niko Cesarovic ${ }^{5}$, Hitendu Dave ${ }^{7}$, Michael Hübler $^{2,7}$, Martin Schweiger $^{2,7}$ \\ ${ }^{1}$ Division of Pediatric Cardiology, Pediatric Heart Center, ${ }^{2}$ Children's Research Center, University Children's Hospital Zurich, Zurich, Switzerland; \\ ${ }^{3}$ Division of Cardiac Anesthesia, Institute of Anesthesiology, University Hospital Zurich, Zurich, Switzerland; ${ }^{4}$ Department of Anesthesia, University \\ Children's Hospital, Zurich, Switzerland; ${ }^{5}$ Division of Surgical Research, University Hospital Zurich, Zurich, Switzerland; ${ }^{6}$ Institute for Veterinary \\ Pathology Vetsuisse Faculty, University of Zurich, Zurich, Switzerland; ${ }^{7}$ Department of Congenital Cardiovascular Surgery, Pediatric Heart Center, \\ University Children's Hospital Zurich, Switzerland \\ Contributions: (I) Conception and design: M Hübler, M Schweiger, B Krüger, T Fleischmann; (II) Administrative support: F Lemme, N Cesarovic; \\ (III) Provision of study materials or patients: All authors; (IV) Collection and assembly of data: A Malbon, H Dave, W Knirsch, B Krüger, M Lipiski, \\ M Sauer, T Fleischmann; (V) Data analysis and interpretation: A Malbon, W Knirsch, B Krüger, T Fleischmann, M Hübler, M Schweiger; (VI) \\ Manuscript writing: All authors; (VII) Final approval of manuscript: All authors. \\ "These authors contributed equally to this work. \\ Correspondence to: Martin Schweiger, MD, PD. Division of Congenital Cardiovascular Surgery, University Children's Hospital, Zurich, Switzerland. \\ Email: martin.schweiger@kispi.uzh.ch.
}

\begin{abstract}
Background: Many valvular pathologies of the heart may be only sufficiently treated by replacement of the valve if a reconstruction is not feasible. However, structural deterioration, thrombosis with thromboembolic events and infective endocarditis are commonly encountered complications over time and often demand a re-operation. In congenital heart disease the lack of small diameter valves with the potential to grow poses additional challenges and limits treatment options to homo- or xenograft implants.

Methods: In this study, a chronic sheep model (24 months follow-up), a self-constructed valved conduit was created out of a tissue engineered (TE) patch (CorMatrix ${ }^{\circledR}$ Cardiovascular, Inc, USA) and implanted in orthotopic right ventricular (RV)-pulmonary artery (PA) position. Thereafter, the sheep were regularly monitored by clinical, laboratory and echocardiographic examinations to evaluate cardiac function and the implanted RV-PA-conduit.
\end{abstract}

Discussion: Here, we summarize the study protocol and our experiences during the perioperative phase and the follow up period and explain how we constructed a valved conduit out of a commercially available TE patch.

Trial registration: License number: $\mathrm{ZH} 284 / 14$.

Keywords: Tissue engineering; pulmonary valve; pulmonary valved conduit; animal model; CorMATRIX

Submitted Jun 05, 2019. Accepted for publication Nov 19, 2019.

doi: $10.21037 /$ jtd.2019.12.70

View this article at: http://dx.doi.org/10.21037/jtd.2019.12.70

\section{Introduction}

While mechanical or biological valve prosthesis are standard of care in adults requiring surgical replacement of heart valves, the surgical repair in children with congenital heart disease (CHD) has specific requirements for valve replacement. This includes small valve diameters with the potential to grow, and specific tissue properties related to age-dependent immunological and degenerative tissue reactions. However, all current used valve substitutes have significant limitations with need for permanent anticoagulation in mechanical prosthesis, potential risk of 
infection, calcification and structural valve degeneration (SVD) in biological prosthesis, leading to reoperation.

In different types of CHD such as common arterial trunk, pulmonary atresia with ventricular septal defect, or patients undergoing a Ross procedure, there is a high demand for a long-lasting connection from the right ventricle (RV) to the pulmonary artery (PA) with an integrated valve. Since the introduction of valved conduits as RV-PA connections in 1966 (1), its use has gained in popularity. Nowadays, the most used RV-PA conduits in CHD are cryopreserved homografts or bovine jugular vein conduits (Contegra ${ }^{\circledR}$ ) (2). While early results for bovine jugular vein conduits are satisfactory with freedom from reoperation of $91 \%$ at 1 year and $84 \%$ at 2 years (3), at long-term follow up, there is a high risk for calcification and SVD which may be related to a stronger immunologic response during childhood (4). While blood-group compatibility seems to play a role for cryopreserved homograft implants (5), the rate of SVD for a homograft is similar to a bioprosthetic valve approximately $30 \%$ after 10 years and $59 \%$ after 15 years (6). Overall, there are no evident differences between those two grafts on long-term follow up (2).

The concept of regenerative medicine using a decellularized porcine small intestinal submucosa extracellular matrix (ECM) biologic scaffold may overcome these limitations. Promising aspects exist with the development of tissue-engineered (TE) heart valves with an appropriate and sustaining hemodynamic profile, low thrombogenic potential and the potential to grow (7). Due to the high demand for valved RV-PA-conduits in congenital heart surgery we evaluated a self-constructed TE valve (CorMatrix ${ }^{\circledR}$ Cardiovascular, Inc., USA) in orthotopic RV to $\mathrm{PA}$ position in a growing large animal model.

Though the use of ovine model for heart valve replacement is not novel (8-10) here we like to share our set-up and highlight differences to other protocols. Likewise, we want to share how we constructed the conduit out of a commercially available patch which could also be useful in clinical practice.

\section{Study rationale}

To test a TE valved conduit in orthotopic pulmonary position which should be physiological, durable and has the potential for growth. In a step-by-step approach, we first self-created a valved conduit out of a commercially available TE patch and developed an adopted surgical implantation technique to lower peri- and postoperative mortality. Second, we established a chronic animal model as proof of feasibility, followed by a proof of concept, resulting in a chronic (2-year) follow-up study. The animal model should clarify, whether the valved pulmonary conduit function determined by echo remains stable and grows during the follow-up period, and finally whether TE patch material will be replaced resp. colonized by host tissue on histological examination after autopsy.

\section{Creating a valved conduit}

A de-cellularized porcine small intestinal submucosa ECM biologic scaffold (Cormatrix ${ }^{\circledR}$ Patch) served as the basis for the RV to PA valved conduit. For the first experiments, the company (CorMatrix ${ }^{\circledR}$ Cardiovascular, Inc., USA) provided uniquely produced valved conduits with a standardized diameter of 20 to $24 \mathrm{~mm}$. Later we changed to a more tailored approach: the conduit was self-constructed out of a commercially available ECM scaffold $(10 \mathrm{~cm} \times 7 \mathrm{~cm}$; fourlayers) according to the size of the pre-operatively measured pulmonary valve annulus (see also operative technique). The longitudinal side was divided in three equal parts mimicking the later created commissures. A ratio of 1:1.2 was established between the conduit diameter and the leaflet height (e.g., for a $20 \mathrm{~mm}$ diameter conduit the leaflets high was $24 \mathrm{~mm}$ ). In each leaflet a "dome" was created from the commissure attachments toward the middle of the leaflet (approximately 2 to $4 \mathrm{~mm}$ in height; Figure $1 A$ ). The leaflet part was then folded (Figure 1B). The attachments of the commissure were enforced with a 6-0 pledged Prolene suture in $\mathrm{Z}$ technique (Figure 1C). Finally, a conduit was formed with a double running suture (Prolene 6-0; Figure 1D). The final length of the valved conduit was tailored accordingly to the length of the resected pulmonary trunk. It was carefully ensured that the conduit was at least a few centimeters longer than the leaflet height to avoid incorporation of the leaflets into the distal suture line. The final conduit may be seen in Figure 2 .

\section{Operative technique}

For the procedure 23 female Swiss White Mountain lambs $(27-38 \mathrm{~kg}, \pm 3.6 \mathrm{SD})$ were anesthetized by board certified veterinary anesthesiologists (outcome may be seen Table 1). Following the establishment of venous access via the internal jugular vein, general anesthesia was induced with a combination of ketamine $(4 \mathrm{mg} / \mathrm{kg}$, iv), midazolam $(0.5 \mathrm{mg} / \mathrm{kg}$, iv) and propofol $(2-5 \mathrm{mg} / \mathrm{kg}$, iv) and, after 

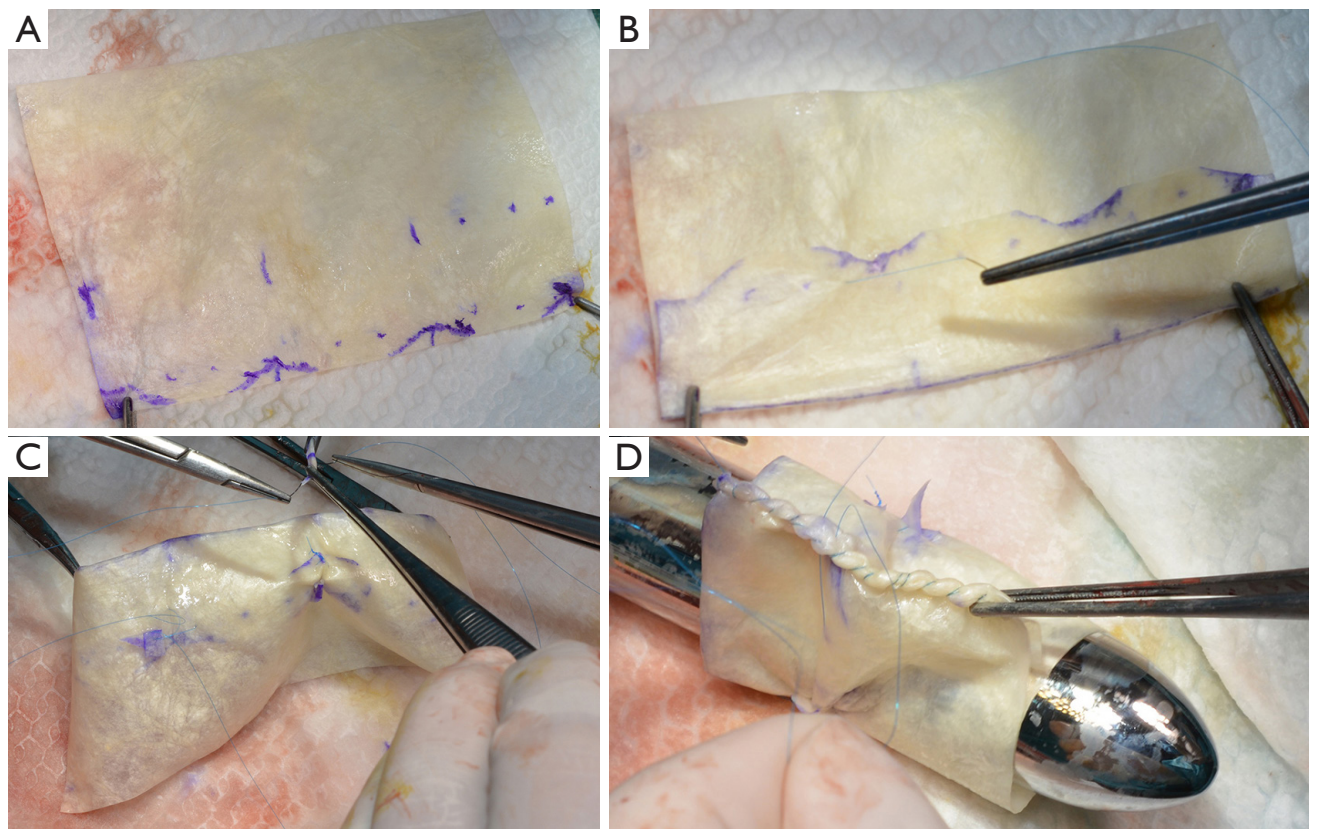

Figure 1 Creation of a valved conduit using a commercially available tissue-engineered (TE) patch. (A) The patch was divided into three equal parts and leaflet height was marked including a doming on top. (B) The patch was folded creating three leaflets. (C) The created commissures were fixed using a 6-0 pledged Prolene suture. (D) Finally, the patch was formed to a conduit and closed using a doublerunning suture line.

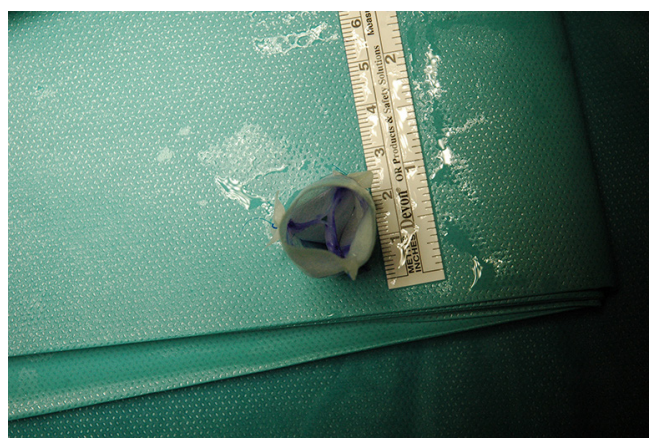

Figure 2 The final conduit created prior to implantation showing the three leaflets.

endotracheal intubation, maintained with isoflurane (1.0-2.0 vol\% endtidal). Perioperative analgesia comprised of a continuous infusion of fentanyl $(10 \mu \mathrm{g} / \mathrm{kg} / \mathrm{h}$, iv $)$ supplemented by boli of fentanyl $(1-2 \mu \mathrm{g} / \mathrm{kg}$, iv) at the discretion of the attending anesthesiologist. Additionally, local anesthesia with ropivacain $(2-3 \mathrm{mg} / \mathrm{kg}$, sc) was performed at the incision site prior to and after surgery. Monitoring included continuous electrocardiogram (ECG), transcutaneous hemoglobin oxygen saturation
$\left(\mathrm{SpO}_{2}\right)$, invasive blood pressure via the femoral artery and an ear artery, central venous pressure via the internal jugular vein, urine output by a bladder catheter and body temperature by a rectal temperature probe. Before start of surgery measurement of lactate dehydrogenase (LDH), hemoglobin (HB), hematocrit (HCT) and white blood cell count (WBC) were performed. During and after surgery, blood gas analysis was performed regularly and serum electrolytes perioperatively supplemented, as required. Lidocaine $1 \%(3 \mathrm{mg} / \mathrm{kg} / \mathrm{h}$, iv) was continuously administered as an antiarrhythmic drug during heart manipulation. Perioperative antibiotic prophylaxis consisted of penicillin $(35,000 \mathrm{IU} / \mathrm{kg}$, iv) and gentamycin $(4 \mathrm{mg} / \mathrm{kg}$, iv) which was continued until postoperative day five. All sheep were provided with a subcutaneous injection of a tetanus vaccine (Tetanus-Serum Intervet, MSD Animal Health; Switzerland). Transesophageal echocardiography (TEE) was performed preoperatively to determine the diameter of the pulmonary annulus and exclude structural heart abnormalities. For the operation the animals were placed in a lateral position. Surgical access was carried out via a left lateral thoracotomy (LLT) in the $4^{\text {th }}$ intercostal 
Table 1 Short-term outcome of the 23 sheep operated on

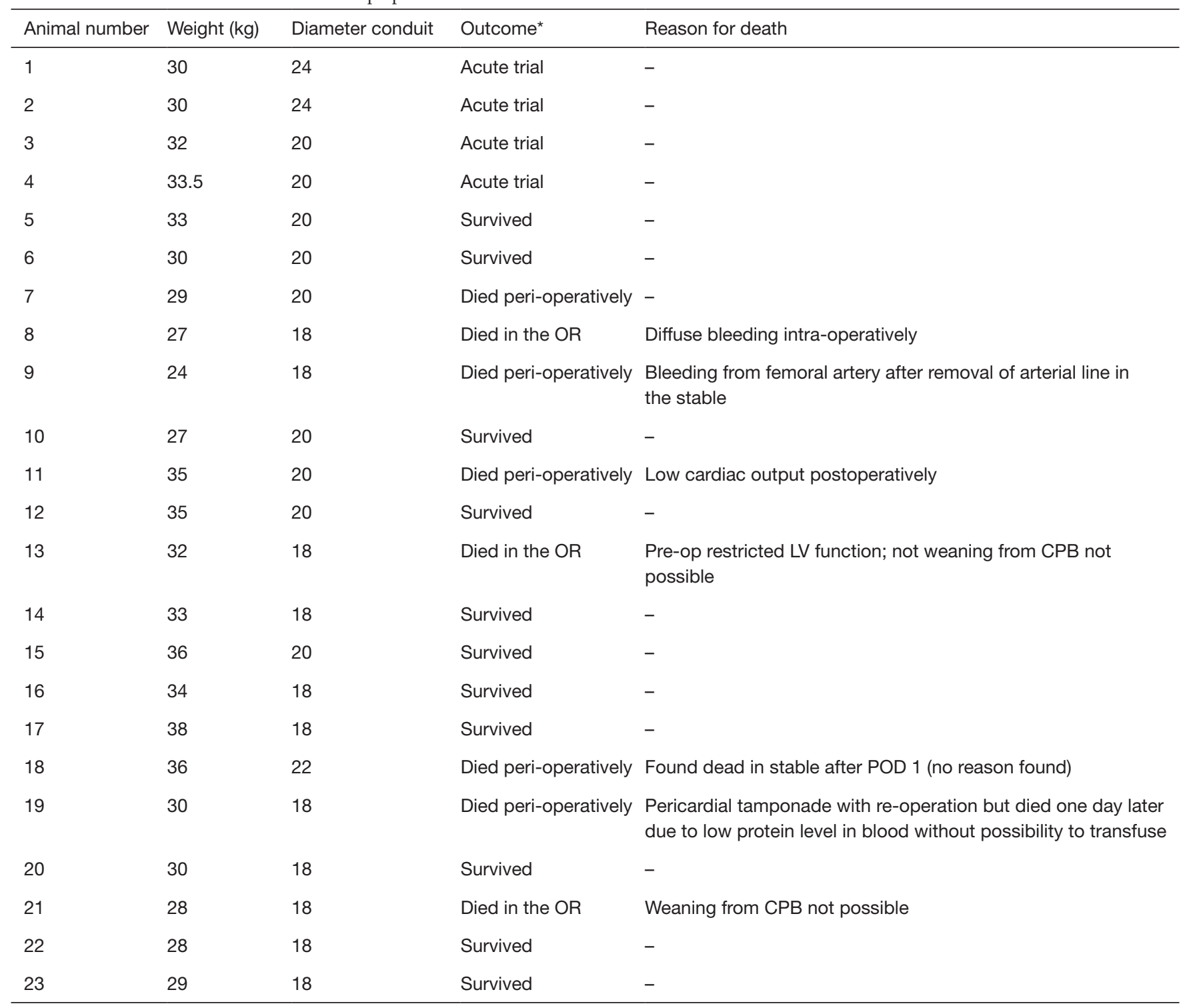

Marked animals survived the operation and peri-operative phase. *, survived: mobilized and send to lawn, died peri-operatively: survived operation and was extubated, mobilization not possible and died within 2 days of operation. LV, left ventricle; POD, postoperative day.

space, without the removal of a rib. The pericardium was opened by protecting the phrenic nerve and stay sutures were placed. The pulmonary trunk was exposed and dissected. The implantation of the valved conduit was performed by use of cardiopulmonary bypass (CPB) without aortic clamping or application of cardioplegia (beating heart). Heparin (5,000 IU, iv) was administered before start of the CPB to establish an activated clotting time (ACT) greater than 300 seconds, which was maintained throughout the CPB period. The bloodless CPB machine priming included an additional 5,000 IU of heparin. The ACT was measured every 15-30 minutes throughout the CPB period and additional heparin boluses were administered as required. Arterial cannulation for CPB was established via the descending aorta using a 14-French cannula and venous cannulation via the right atrium using a single stage 30-French cannula. Once on $\mathrm{CPB}$, mild hypothermia $\left(35^{\circ} \mathrm{C}\right)$ was targeted and the native pulmonary valve and the complete pulmonary trunk were resected (Figure 3). Stay sutures were placed in the native pulmonary valve anulus at 


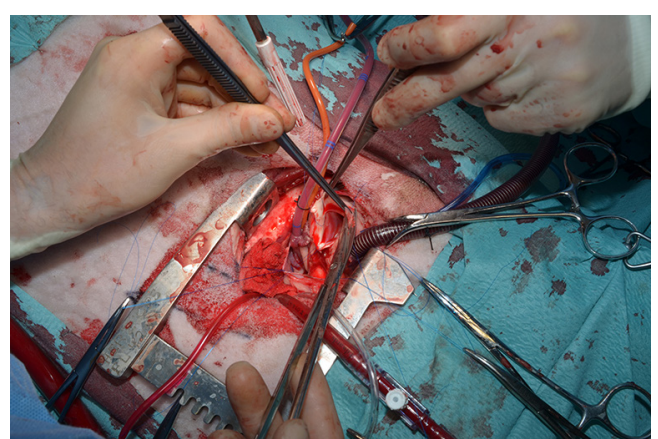

Figure 3 The native pulmonary valve leaflets as well as the complete pulmonary trunk were resected.

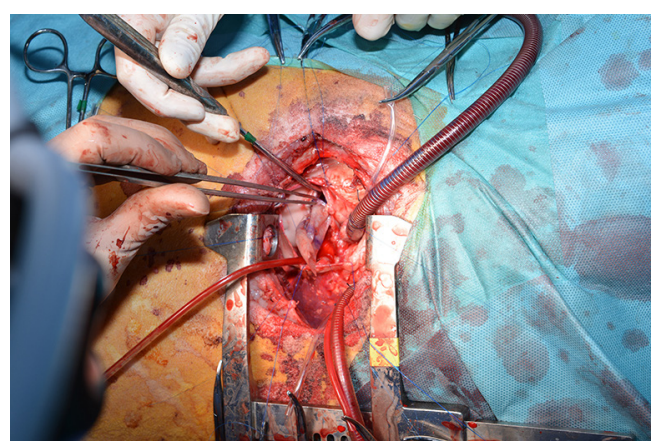

Figure 4 After correct orientation the valved conduit was implanted using a running suture.

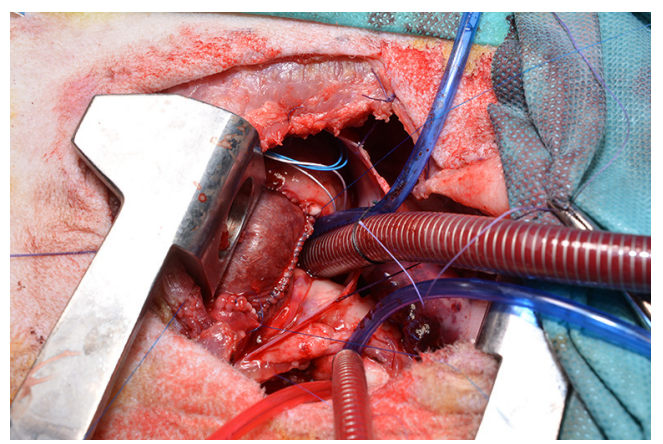

Figure 5 The image shows the implanted valved conduit with the suture line of the conduit pointing cranial-aortal for better bleeding control.

the edges of the three commissures to preserve the original valve orientation. The substitute was then implanted using a running suture (6-0 polypropylene) following the origin of the explant line (Figure 4) and orientated according to the stay sutures and the mounting of the leaflets within the conduit to achieve an accurate geometry. The suture line of the conduit pointed cranial/aortal for better bleeding control (Figure 5). After completing the distal anastomosis with a running suture (6-0 Prolene) the animals were weaned from CPB. Protamine (2,500 IU in $100 \mathrm{~mL} \mathrm{NaCl}$, iv) was administered under close observation of ACT until levels were below 300 seconds. Finally, after controlling major sources of bleeding, a chest tube was placed, and chest closure performed followed by a postoperative TEE. The final result of the implantation is available in Video 1.

\section{Postoperative care}

After surgery, the sheep were positioned in dorsal recumbency and closely monitored during recovery from anesthesia. All animals received external heat support (warming blanket, Bair Hugger ${ }^{\mathrm{TM}}$ ), warmed fluids and electrolyte supplementation (e.g., $\mathrm{Mg}, \mathrm{Ca}, \mathrm{K}$ ) as needed. The thoracic drainage tube was evacuated regularly and removed shortly before removal of the endotracheal tube. After extubation, 100\% oxygen was provided via a nasal mask. Each sheep was kept in the recovery area until respiratory rate, $\mathrm{SpO}_{2}$ and core body temperature were within the normal range, and the animal was able to maintain pink mucous membranes under ambient air conditions. For post-operative analgesia methadone $(0.2 \mathrm{mg} / \mathrm{kg}$, iv) was administered up to 3 days and metamizole (20 mg/kg, iv) up to 5 days. Carprofen ( $4 \mathrm{mg} / \mathrm{kg}$, iv) was given at the end of the operation and once per day for up to 7 days, as needed.

Before transporting the animals back to the stable, both arterial catheters, one of the two jugular catheters and the urinary catheter were removed. The jugular venous catheter was protected with a bandage and remained in place for 5 days, to allow for regular blood electrolyte analysis and intravenous drug administration. In the stable, the animals were kept in a single compartment for intensive care, but with nose-to-nose contact to companions. A warming lamp was used to facilitate recovery. Postoperative care continued until the animals were able to stand without ataxia and were starting to eat and drink.

All animals received acetylsalicylic acid $(100 \mathrm{mg}$, po, once per day) for a total of 5 days. Antibiotics (Penicillin 35,000 IU/kg twice a day, iv and gentamycin $4 \mathrm{mg} / \mathrm{kg}$ once a day, iv) were administered for up to 5 days after surgery. Intensive care was provided for at least 5 days following surgery, depending on the state of the animal. Each sheep received 
at least twice daily a full clinical examination, including physical exam, observation of surgical site, assessment of attitude, activity, appetite, fecal and urine production, vital parameters (body temperature, pulse rate, respiratory rate), heart auscultation and assessment of potential signs of pain or reduced well-being. Blood electrolyte analysis and ACT were performed twice per day or when deemed necessary. If an animal appeared weak or lethargic, suggesting cardiovascular or respiratory compromise, further examinations were performed including echocardiography under general anesthesia.

From the fifth postoperative day on, the animals received a full clinical examination once daily. Around this time, all sheep usually were in a stable condition and could be resocialized with their companions, without further need of medication or intensive care.

In every animal a routine follow-up echocardiography was performed in general anesthesia within the first month after surgery.

After verification of functionality of the conduit, all animals were transported back to the farm of the vendor for long-term husbandry and surveillance (clinical check at least three time a week). Thereafter, the animals were brought back only for the scheduled routine follow-up controls or in case of pathological signs during the routine clinical examinations on the farm.

\section{Echo protocol}

Transesophageal (TEE) and transthoracic (TTE) twodimensional Colour-Doppler echocardiography were used to evaluate cardiac function and the structure and functionality of the implanted RV-PA-conduit intraoperatively and during follow-up controls. All examinations were realized under general anesthesia and were performed by pediatric and/or adult TEE/TTEcertified personnel on a Philips iE33 ultrasound machine (Philips Center, Amsterdam, The Netherlands) by use of a 7-2 MHz multiplane echo probe (Philips X7-2t) for TEE and a 5-1 MHz echo probe (Philips S5-1) for TTE. We evaluated the morphologic (thickness, mobility, and potential calcification of valve leaflets) and functional (valve stenosis and regurgitation) appearance of the valved RVPA-conduit. This included measurements of the diameter at a sub-valvular, valvular and supravalvular level and of the maximal systolic and mean pressure gradient using pulsed and continuous-wave Doppler. Valvular regurgitation of the pulmonary conduit was quantified using Colour Doppler and retrograde directed pulsed-wave Doppler signals in the main PA and main side branches. The aortic valve was monitored in comparison to the valved pulmonary conduit. Right ventricular (RV) function was qualitatively evaluated by eyeballing and categorized as normal, moderately or severely impaired. The RV size was evaluated by measuring the diameter of the tricuspid valve annulus, the inflow tract, ventricle, and outflow tract in a standardized view in TTE (Figure 6A,B). 3-D Echocardiography to reconstruct the pulmonary valve was done as well (Figure $6 C$ ). Tricuspid valve regurgitation was evaluated by Colour Doppler.

\section{Follow-up protocol}

Follow-up controls included a clinical, laboratory and echocardiographic examination and were scheduled at regular time intervals: once within the first post-operative month, at 3, 6, 9, 12, 18 and 24 months. For each of these controls, the animals were transferred back from the farm to the Division of Surgical Research. Growth and thriving were monitored by body weight. Laboratory checks for hemolysis and infection included measurements of lactate dehydrogenase (LDH), hemoglobin (HB), hematocrit (HCT) and white blood cell count (WBC). At the end of the study period, the RV-PA-conduit was evaluated for calcification by a CT scan followed by thorough pathological evaluation after scarification of the animal. Macroscopically cardiac examination was performed both in-situ and following removal of the intact heart. The chambers of the heart were opened along the path of blood flow to allow a full visual assessment. The PA (both native and conduit) was opened longitudinally, cutting at the valve commissure to preserve the integrity of the leaflets. All steps were photographic documented including a video clip of the still beating heart when the conduit was free from adhesions. The entire heart was fixed in 10\% neutral buffered formalin before cutting in the tissues for processing. Each valve was bisected so that each tissue section included the center of the valve leaflet and the proximal and distal junctions of the conduit. After a minimum of 24 hours fixation, tissues were routinely processed through a graded series of alcohol, embedded in paraffin and sectioned at $2 \mu \mathrm{m}$ for haematoxylin and eosin staining.

For a subset of the valves, consecutive sections were mounted on standard or positively charged slides and used for special staining or immunohistology respectively. The panel included the special stains Masson's trichrome, Elastic Van Gieson, and Von Kossa, and the immunohistological 

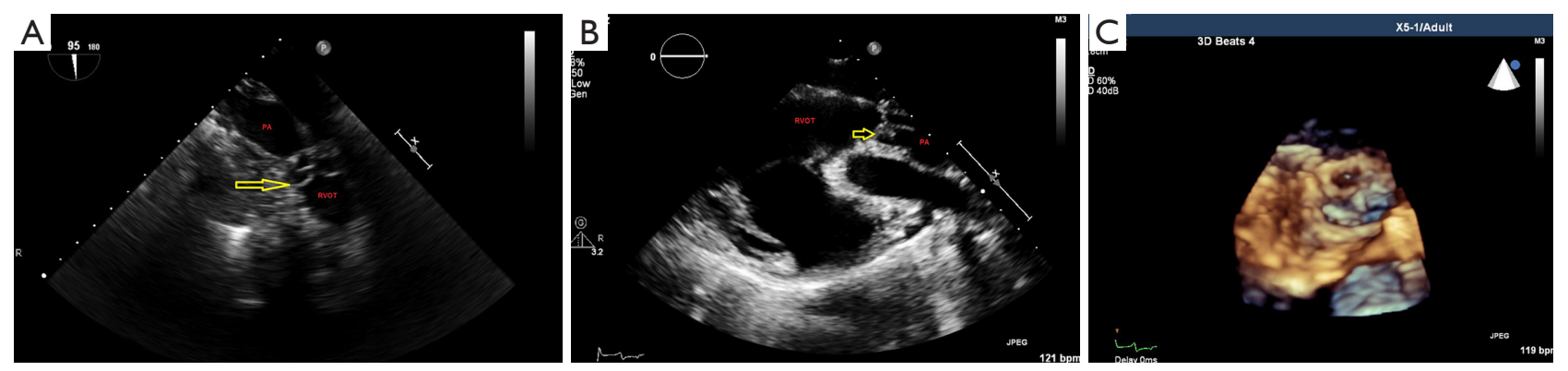

Figure 6 For routine follow-up visits the valved conduit was evaluated by means of transesophageal and transthoracic echocardiography (TTE) using standardized views. (A-C) Here an example of a 1-year follow-up examination; yellow arrow pointing toward pulmonary valve leaflets. The images show TTE for measuring RV size at the inflow tract, ventricle, and outflow tract as well as diameter of the tricuspid valve annulus. RVOT, right ventricular outflow tract; PA, pulmonary artery.

markers alpha smooth muscle actin (smooth muscle), CD31 (endothelial cells), Ki-67 (proliferation marker), caspase 3 (apoptosis), and CD68 (cells of monocyte lineage).

\section{Comments}

So far, most studies in animals using the Comatrix patch as complete or partial valve substitute have a mid-term follow up of three to eight months (11-14). There are hints that more long-term data from animal trials are needed as there seems to be doubt about mid-term outcome using this material for valve substitutes (15). Therefore, we established a growing animal model to evaluate a TE valved conduit. The use of ovine model for heart valve replacement has been reported but mostly in adult sheep (8) or a shorter follow up $(9,10)$. To the authors knowledge this is the first description of a lamb model aimed on a long-term outcome reaching a follow up time of 2 years. Likewise, importantly, we were able to self-construct the valved conduit out of a commercially available patch already used in clinical practice.

From our experience and study set-up we would like to share some key findings to reinforce the importance and validity of detailed study protocols.

(I) The advantage of a sheep model relies on anatomical and hemodynamic similarities compared to human physiology, e.g., the molecular basis of cardiac contraction, the coronary anatomy and the hemodynamic profile of the pulmonary arteries (16). Compared to a human, structural changes and calcification of a cardiac valve (primary outcome parameter) occurs much earlier in an ovine. Because 1 year of a sheep life roughly equals 7 years of a human's life 'long-term' outcome results may be acquired in shorter time periods (ratio 1:7);

(II) From other pre-clinical experiments using off-theshelf de-cellularized TE patches $(17,18)$ as aortic valve leaflet (19) we learned that the potential benefits of a thoracotomy outweighed those of a sternotomy, especially when considering a chronic study. According to our experience using a LLT via the fourth intercostal space (i) offers good exposure to the pulmonary trunk and the pulmonary valve, (ii) allows reliably the cannulation of the descending aorta and the right atrium via the right auricle and (iii) promotes early extubation and sufficient spontaneous breathing as postoperative pain is diminished in these animals normally lying on the sternum;

(III) The exposure of the ascending aorta, clamping and infusion of cardioplegia is technically challenging in sheep due to the short size of the ascending aorta. We have experienced that the avoidance of cardioplegia overweighs the inconvenience to perform the valve implantation in beating heart conditions;

(IV) Hemolysis is one of the potentially serious complications of prosthetic heart valves and is usually associated with structural deterioration. Only sparse reference values are available in the sheep model. To gain more data about this important issue, we suggest to monitor the values of relevant blood hemolysis parameters ( $\mathrm{LDH}, \mathrm{Hb}$ ) over time;

(V) A proper evaluation of the implanted valve and the heart function requires a combined transesophageal and transthoracic examination which can be done in a short-duration general anesthesia. During 
the scheduled examinations we did not lose any animal due to hemodynamic impairment caused by anesthesia. Nevertheless, we recommend the examination being performed by certified and experienced personnel to obtain good quality imaging in the shortest amount of time to limit the duration of general anesthesia.

We believe that failure to make a detailed study protocol for such extensive animal experiments may result in important limitations at the end of the study and effect animal welfare in a negative way. Our experiences and comments may help other groups using a sheep model in cardiovascular disease research to improve survival rates and increase animal welfare.

So far, most studies in animals using the Comatrix patch as complete or partial valve substitute have a three to 6 months follow-up. We believe that more long-term data (>12 months follow-up) is needed.

\section{Limitation}

So far there is no sufficient evidence that CorMatrix ${ }^{\circledR}$ tissue can grow. Some recent clinical studies show that the patches are prone to calcification and failure. The used patches here were not seeded with live autologous cells. Although it would have been interesting to test conduits with smaller diameters none of our animal had a pulmonary valve annulus below 18 $\mathrm{mm}$ and we did not want to undersize the conduit.

\section{Acknowledgments}

Funding: The acute animal experiments were supported by a research grant of Cormatrix (CorMatrix Cardiovascular, Inc., USA). Furthermore, we were provided with the patch material for the experiments.

\section{Footnote}

Conflicts of Interest: The authors have no conflicts of interest to declare.

Ethical Statement: The authors are accountable for all aspects of the work in ensuring that questions related to the accuracy or integrity of any part of the work are appropriately investigated and resolved. Animal housing and experimental protocols were approved by the Cantonal Veterinary Office, Zurich, Switzerland (license no. ZH 284/14), and were in accordance with the Swiss Animal Protection Law.
Housing and experimental procedures were also conformed to European Directive 2010/63/EU of the European Parliament, and of the Council of 22 September 2010 on the Protection of Animals used for Scientific Purposes and to the Guide for the Care and Use of Laboratory Animals (2010/63/ EU, 2010; Balinger et al., 2011).

Open Access Statement: This is an Open Access article distributed in accordance with the Creative Commons Attribution-NonCommercial-NoDerivs 4.0 International License (CC BY-NC-ND 4.0), which permits the noncommercial replication and distribution of the article with the strict proviso that no changes or edits are made and the original work is properly cited (including links to both the formal publication through the relevant DOI and the license). See: https://creativecommons.org/licenses/by-nc-nd/4.0/.

\section{References}

1. Ross DN, Somerville J. Correction of pulmonary atresia with a homograft aortic valve. Lancet 1966;2:1446-7.

2. Poinot N, Fils JF, Demanet H, et al. Pulmonary valve replacement after right ventricular outflow tract reconstruction with homograft vs Contegra(R): a case control comparison of mortality and morbidity. J Cardiothorac Surg 2018;13:8.

3. Dave H, Dodge-Khatami A, Kadner A, et al. Modified technique for heterotopic implantation of a right ventricular outflow tract conduit. Ann Thorac Surg 2006;81:2321-3.

4. Baskett RJ, Ross DB, Nanton MA, et al. Factors in the early failure of cryopreserved homograft pulmonary valves in children: preserved immunogenicity? J Thorac Cardiovasc Surg 1996;112:1170-8; discussion 1178-9.

5. Christenson JT, Sierra J, Colina Manzano NE, et al. Homografts and xenografts for right ventricular outflow tract reconstruction: long-term results. Ann Thorac Surg 2010;90:1287-93.

6. Rahimtoola SH. Choice of prosthetic heart valve in adults an update. J Am Coll Cardiol 2010;55:2413-26.

7. Schweiger M, Dave H, Schmiady M, et al. The Search for the Optimal Right Ventricular Outflow Tract Conduit. J Heart Cardiovasc Med 2018;1:3.

8. Bennink G, Torii S, Brugmans M, et al. A novel restorative pulmonary valved conduit in a chronic sheep model: Midterm hemodynamic function and histologic assessment. J Thorac Cardiovasc Surg 2018;155:2591-601.e3.

9. Kluin J, Talacua H, Smits AI, et al. In situ heart valve tissue 
engineering using a bioresorbable elastomeric implant From material design to 12 months follow-up in sheep. Biomaterials 2017;125:101-17.

10. Quinn RW, Hilbert SL, Bert AA, et al. Performance and morphology of decellularized pulmonary valves implanted in juvenile sheep. Ann Thorac Surg 2011;92:131-7.

11. Mosala Nezhad Z, Poncelet A, Fervaille C, et al. Experimental Aortic Valve Cusp Extension with CorMatrix in a Porcine Model. Thorac Cardiovasc Surg 2017;65:206-10.

12. Boni L, Chalajour F, Sasaki T, et al. Reconstruction of pulmonary artery with porcine small intestinal submucosa in a lamb surgical model: Viability and growth potential. J Thorac Cardiovasc Surg 2012;144:963-9.e1; discussion 969.

13. Miller JR, Henn MC, Lancaster TS, et al. Pulmonary Valve Replacement With Small Intestine SubmucosaExtracellular Matrix in a Porcine Model. World J Pediatr Congenit Heart Surg 2016;7:475-83.

14. Zafar F, Hinton RB, Moore RA, et al. Physiological Growth, Remodeling Potential, and Preserved Function of a Novel Bioprosthetic Tricuspid Valve: Tubular

Cite this article as: Knirsch W, Krüger B, Fleischmann T, Malbon A, Lipiski M, Lemme F, Sauer M, Cesarovic N, Dave H, Hübler M, Schweiger M. Establishing a pre-clinical growing animal model to test a tissue engineered valved pulmonary conduit. J Thorac Dis 2020;12(3):1070-1078. doi: 10.21037/ jtd.2019.12.70
Bioprosthesis Made of Small Intestinal Submucosa-Derived Extracellular Matrix. J Am Coll Cardiol 2015;66:877-88.

15. Padalino MA, Castaldi B, Fedrigo M, et al. Porcine Intestinal Submucosa (CorMatrix) for Semilunar Valve Repair in Children: A Word of Caution After Midterm Results. Semin Thorac Cardiovasc Surg 2016;28:436-45.

16. DiVincenti L, Jr., Westcott R, Lee C. Sheep (Ovis aries) as a model for cardiovascular surgery and management before, during, and after cardiopulmonary bypass. J Am Assoc Lab Anim Sci 2014;53:439-48.

17. Weber B, Dijkman PE, Scherman J, et al. Off-the-shelf human decellularized tissue-engineered heart valves in a non-human primate model. Biomaterials 2013;34:7269-80.

18. Hoerstrup SP, Cummings Mrcs I, Lachat M, et al. Functional growth in tissue-engineered living, vascular grafts: follow-up at 100 weeks in a large animal model. Circulation 2006;114:I159-66.

19. Schweiger M, Knirsch W, Cesarovic N, et al. Surgical technique: establishing a pre-clinical large animal model to test aortic valve leaflet substitute. J Thorac Dis 2016;8:3733-8. 